\title{
Análise da influência dos hormônios sexuais na Doença de Alzheimer: revisão integrativa de literatura
}

\author{
Analysis of the influence of sex hormones on Alzheimer's Disease: an integrative literature review \\ Análisis de la influencia de las hormonas sexuales en la enfermedad de Alzheimer: revisión \\ integradora de la literatura
}

Bárbara Souza Fonseca1*, John Klysman Araujo², João Pedro Moraes Borges ${ }^{1}$, Letícia Damasceno Janhaki Mota ${ }^{3}$, Luíza Vitória Evangelista de Miranda ${ }^{3}$, Tenylle Botelho Fernandes ${ }^{1}$, Tereza Paula Pereira Santos ${ }^{1}$, Sabrina Suellem Soares Barbosa ${ }^{1}$, Mariane Costa Santos ${ }^{1}$, Clarissa Leal Silva e Souza ${ }^{1}$.

\begin{abstract}
RESUMO
Objetivo: Analisar a influência dos hormônios sexuais na fisiopatologia e predisposição da Doença de Alzheimer, além dos seus benefícios neuroprotetores. Métodos: Trata-se de uma revisão integrativa da literatura e foi realizada nas bases de dados PubMed, Biblioteca Virtual em Saúde (BVS) e Scientific Electronic Libray Online (SciELO), utilizando palavras chaves incluídas nos Descritores em Ciências da Saúde (DeCS), em suas variantes na língua inglesa e portuguesa, nas seguintes combinações utilizando o operador booleano AND: "Doença de Alzheimer AND Hormônios Sexuais" e "Alzheimer Disease AND Sex Hormones". Resultados: Foram selecionados 18 artigos para revisão. A partir da leitura dos artigos foi observado as diferenças sexuais na Doença de Alzheimer, mediadas principalmente pelos hormônios esteroidais. Verificou-se a atividade neuroprotetora desses hormônios contra processos inflamatórios no Sistema Nervoso Centra Considerações finais: Os estudos mostram a participação dos hormônios sexuais na fisiopatologia da doença de Alzheimer. As alterações desses esteroides influenciam em diferentes sítios, como: proteínas, organelas, células neuronais e da glia, resultando no processo neurodegenerativo que caracteriza a doença. Portanto, considera-se um campo promissor em pesquisas voltadas para a terapia dessa demência, inclusive destacando quais seriam mais benéficas para cada sexo.
\end{abstract}

Palavras-chave: Doença de Alzheimer, Hormônios sexuais, Diferenças sexuais, Neuroprotetores.

\begin{abstract}
Objective: To analyze the influence of sex hormones on the pathophysiology and predisposition of Alzheimer's Disease, in addition to their neuroprotective benefits. Methods: This is an integrative literature review, and was carried out in the PubMed, Virtual Health Library (BVS) and Scientific Electronic Library Online (SciELO) databases, using key words included in the Health Sciences Descriptors (DeCS), in its variants in English and Portuguese, in the following combinations using the Boolean operator "AND": "Alzheimer's Disease AND Sex Hormones and Alzheimer's Disease AND Sex Hormones". Results: 18 articles were selected for review. From reading the articles, sex differences in Alzheimer's disease were observed, mainly mediated by steroid hormones. Furthermore, the activity of these hormones as neuroprotectors against inflammatory processes in the central nervous system is verified. Conclusion: Studies show the participation of sex hormones in the pathophysiology of Alzheimer's disease. Changes in these steroids influence different sites, such as: proteins, organelles, neuronal and glial cells, resulting in the neurodegenerative process that characterizes the disease. Therefore, it is considered a promising field in research aimed at the therapy of this dementia, including highlighting which would be more beneficial for each sex.
\end{abstract}

Keywords: Alzheimer's disease, Sex hormones, Sex differences, Neuroprotectors.

\section{RESUMEN}

Objetivo: Analizar la influencia de las hormonas sexuales en la fisiopatología y predisposición de la enfermedad de Alzheimer, además de sus beneficios neuroprotectores. Métodos: Revisión integradora de la literatura, y se realizó en las bases de datos PubMed, Virtual Health Library (BVS) y Scientific Electronic Library Online (SciELO), utilizando palabras clave incluidas en los Health Sciences Descriptors (DeCS), en sus variantes en Inglés y portugués, en las siguientes combinaciones utilizando el operador booleano "Y": "Enfermedad de Alzheimer Y hormonas sexuales y Enfermedad de Alzheimer Y hormonas sexuales". Resultados: se seleccionaron 18 artículos para revisión. Al leer los artículos, se observaron diferencias de sexo en la enfermedad de Alzheimer, principalmente mediadas por hormonas esteroides. Además, estas hormonas tienen actividad como neuroprotectores frente a procesos inflamatorios en el sistema nervioso central. Conclusión: Los estudios muestran la participación de las

\footnotetext{
${ }^{1}$ Faculdade de Saúde Santo Agostinho (FASA), Vitória da Conquista - BA. *E-mail: barbarasfonseca24@gmail.com

2 Universidade Estadual do Sudoeste da Bahia (UESB), Vitória da Conquista - BA.

${ }^{3}$ Faculdade de Saúde Santo Agostinho (FASA), Itabuna - BA.
} 
hormonas sexuales en la fisiopatología de la enfermedad de Alzheimer. Los cambios en estos esteroides influyen en diferentes sitios, tales como: proteínas, orgánulos, células neuronales y gliales, dando como resultado el proceso neurodegenerativo que caracteriza a la enfermedad. Por tanto, se considera un campo prometedor en la investigación orientada a la terapia de esta demencia, destacando cuáles serían más beneficiosas para cada sexo.

Palabras clave: Enfermedad de Alzheimer, Hormonas sexuales, Diferencias sexuales, Neuroprotectores.

\section{INTRODUÇÃO}

O aumento da expectativa de vida trouxe consigo o aumento na prevalência dos diagnósticos de doenças neurodegenerativas, inclusive a Doença de Alzheimer (DA). O número global de pessoas que vivem com a DA, em 2016, foi de 43,8 milhões (NICHOLS E, et al., 2019). De acordo com a Organização Mundial da Saúde (2021) em 2019 a Doença de Alzheimer e outras demências representaram cerca de 28352 anos de vida perdidos ajustados por incapacidade (indicador Dalys), sendo que no mesmo ano contabilizou-se em todo o mundo 1,6 milhões de morte devido a demências, incluindo a Doença de Alzheimer (WORLD HEALTH ORGANIZATION, 2021).

A DA está relacionada com um declínio cognitivo progressivo, sendo marcada pela perda da memória e mudança de comportamento, que ocorre como consequência do processo neurodegenerativo que envolve a fisiopatologia da doença (PRINCE M, 2013). As características patológicas da DA envolvem a deposição da proteína beta amiloide $(A \beta)$, formando placas senis extracelulares, além da emaranhados neurofibrilares intracelulares da proteína Tau, que se encontram anormalmente fosforiladas. Observa-se ainda alterações nos espaços perivasculares dos pacientes acometidos, que culminam na menor depuração da proteína beta amiloide (TEJEDOR NV, et al., 2021).

Avaliando as diferenças epidemiológicas relacionadas ao sexo, pode-se observar um maior risco para DA na população feminina. Essa diferença pode estar associada a maior expectativa de vida das mulheres (NICHOLS E, et al., 2019). Entretanto, estudos apontam que os hormônios sexuais, como o estrogênio, são neuroprotetores. Assim, deficiência estrogênica pode intensificar ou precipitar o processo da DA nas mulheres (ZÁRATE S, et al., 2017; CUI J, et al., 2013).

Os hormônios sexuais, progesterona, estradiol, estrona, 3a-androstanodiol e testosterona, envolvidos nas funções cerebrais, são capazes de aumentar os níveis de ATP e a respiração mitocondrial (GRIMM A, et al., 2015). Por outro lado, a redução na disponibilidade dos hormônios sexuais coincide com o processo de envelhecimento, como o que ocorre na perimenopausa e na menopausa. A redução dos esteroides sexuais femininos é responsável por alterações diversas no organismo, inclusive na cognição. Tal afirmação pode ser uma suposta explicação para maior incidência e prevalência da DA no sexo feminino (BRINTON RD, et al., 2015).

Associada as ações hormonais, algumas particularidades genéticas podem contribuir para a maior predisposição da DA no sexo feminino. Mulheres que carregam apolipoproteina $E$ (ApoE) com alelo e4 desenvolvem a DA com maior facilidade. Contudo, o mesmo não se observa no sexo masculino, onde a ApoE4 não se comporta como um fato estimulador para doença (ALTMANN A, et al., 2014).

Sabe-se que a demência acomete progressivamente a qualidade de vida do paciente, além de afetar os familiares ao seu redor, já que a pessoa adoecida se torna cada vez mais dependente (RODRIGUES TQ, et al., 2020). Por isso, estudos que investigam a fisiopatologia da DA podem contribuir para investigação dos possíveis meios de atuação contra a doença, visando ao menos retardar o início ou controlar sua progressão. Assim, pesquisas voltadas para atuação dos hormônios sexuais na DA tem um papel promissor em uma possível terapia (GIRARD R, et al., 2017). Diante do exposto, esse estudo teve como objetivo avaliar a influência dos hormônios sexuais na resposta fisiopatológica e na predisposição da DA, além dos seus benefícios neuroprotetores.

\section{MÉTODOS}

Este estudo é uma revisão integrativa de literatura baseada em artigos originais publicados de 2016 a 2020. Afim de alcançar os objetivos traçados foi elaborada a seguinte pergunta norteadora: Como os hormônios sexuais influenciam na fisiopatologia e predisposição da Doença de Alzheimer? 
Em abril e maio de 2021, realizou-se a busca pelos artigos em bancos de dados eletrônicos, PubMed, Biblioteca Virtual em Saúde (BVS) e Scientific Electronic Libray Online (SciELO), utilizando palavras chaves incluídas nos Descritores em Ciências da Saúde (DeCS), em suas variantes na língua inglesa e portuguesa, nas seguintes combinações utilizando o operador booleano AND: "Doença de Alzheimer AND Hormônios Sexuais" e "Alzheimer Disease AND Sex Hormones".

Como critérios de inclusão foram selecionados artigos originais com texto integral disponível em formato eletrônico, gratuito, sendo compatível com no mínimo um dos objetivos da pesquisa e redigido nos seguintes idiomas: português, inglês ou espanhol e com recorte temporal de 2016 a 2020. Excluíram-se artigos de revisão de literatura, artigos de opinião, relatos de caso, cartas ao editor e artigos duplicados. Ademais, foram descartados estudos cujo objeto central se distanciava da discussão sobre a influência dos hormônios sexuais na doença de Alzheimer e que foram publicados fora do recorte temporal supracitado. Após realizada a busca nas bases de dados e avaliado os artigos de acordo com os critérios de inclusão e exclusão, foram selecionados 99 artigos para revisão. Após a leitura dos títulos e resumos foram separados 17 artigos. Os 17 artigos foram lidos na íntegra e usados para abordagem do assunto (Figura 1).

Figura 1 - Fluxograma representativo da metodologia de inclusão dos artigos neste estudo.
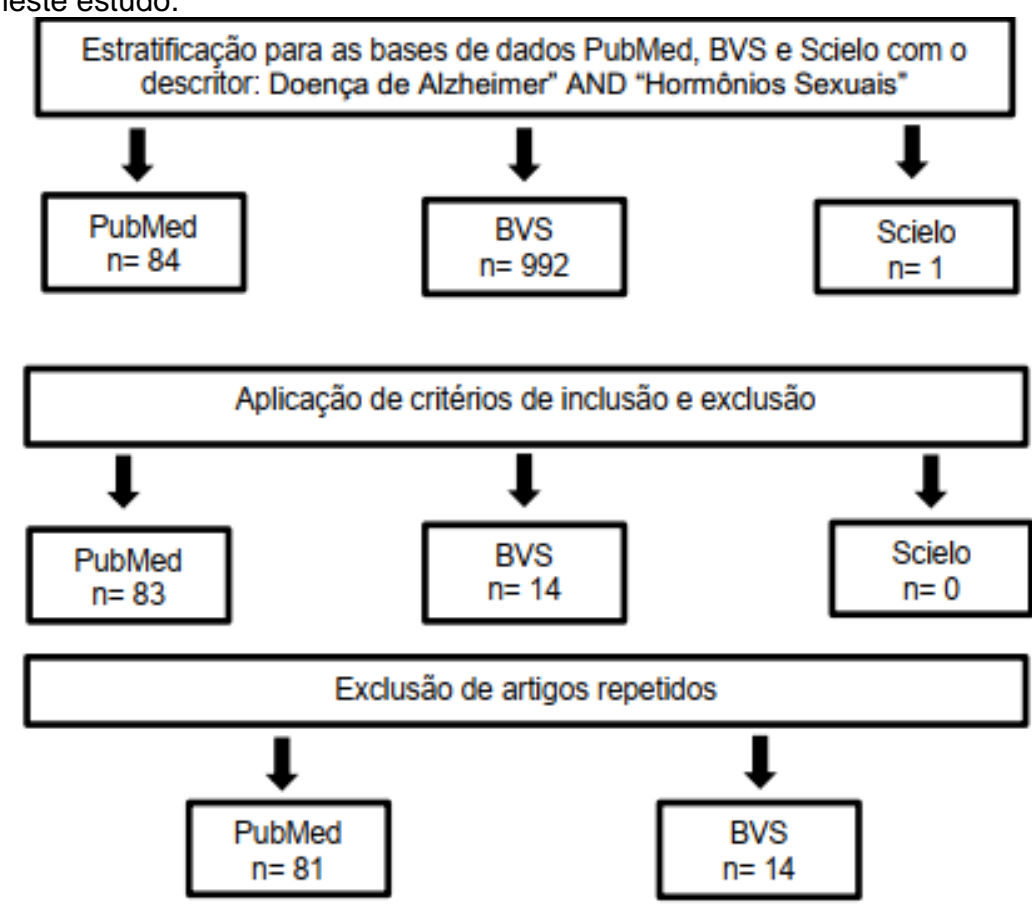

Exclusão dos artigos que não contemplam o objetivo do estudo

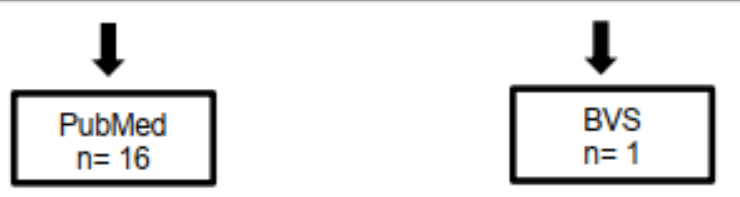

Número de artigos considerados para leitura na integra: 17

Fonte: Fonseca BS, et al., 2021.

\section{RESULTADOS}

Dentre os 17 artigos selecionados e incluídos na revisão integrativa a partir da leitura na íntegra, observouse que todos artigos foram escritos no idioma da língua inglesa e utilizaram experimentos in vivo, in vitro e amostra de dados demográficos (Quadro 1). 


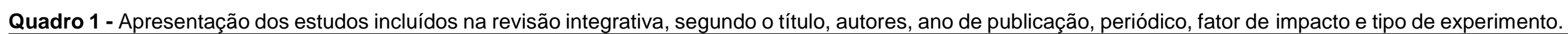

\begin{tabular}{|c|c|c|c|c|c|}
\hline Ano & Autores & Título do artigo & Periódico & Fator de impacto & Tipo de experimento \\
\hline 2020 & $\begin{array}{l}\text { DUARTE AC, et } \\
\text { al. }\end{array}$ & $\begin{array}{l}\text { Idade, hormônios sexuais e ritmo circadiano regulam a } \\
\text { expressão de necrófagos beta-amilóide no plexo coróide }\end{array}$ & $\begin{array}{l}\text { International Journal of } \\
\text { Molecular Sciences }\end{array}$ & 5,9 & In vivo \\
\hline 2019 & KHAN M, et al. & $\begin{array}{l}\text { O 17ß-estradiol modula SIRT1 e interrompe o } \\
\text { comprometimento cognitivo mediado por estresse } \\
\text { oxidativo em um modelo de camundongo com } \\
\text { envelhecimento masculino }\end{array}$ & Cells & 63 & In vivo \\
\hline 2017 & $\begin{array}{l}\text { HONMA N, et } \\
\quad \text { al. }\end{array}$ & $\begin{array}{l}\text { Fatores relacionados ao estrogênio no lobo frontal de } \\
\text { pacientes com doença de Alzheimer e a importância do } \\
\text { índice de massa corporal }\end{array}$ & Scientific reports & 7,1 & In vitro \\
\hline 2016 & $\begin{array}{l}\text { BANGASSER } \\
\quad \text { D, et al. }\end{array}$ & $\begin{array}{l}\text { A superexpressão do fator de liberação de corticotropina } \\
\text { dá origem a diferenças sexuais na sinalização } \\
\text { relacionada à doença de Alzheimer }\end{array}$ & Mol Psychiatry & 6,7 & In vivo \\
\hline 2018 & $\begin{array}{l}\text { BROESTL L, et } \\
\text { al. }\end{array}$ & $\begin{array}{l}\text { Os estágios do ciclo ovariano modulam alterações } \\
\text { cognitivas e da rede cerebral relacionadas ao Alzheimer } \\
\text { em camundongos fêmeas }\end{array}$ & eNeuro & 5,3 & In vivo \\
\hline 2018 & $\begin{array}{l}\text { SNYDER B, et } \\
\text { al. }\end{array}$ & $\begin{array}{l}\text { Os andrógenos modulam os efeitos da hipóxia } \\
\text { intermitente crônica no cérebro e no comportamento }\end{array}$ & Horm Behav & 6,4 & In vivo \\
\hline 2020 & DUONG P, et al. & $\begin{array}{l}\text { Resultados neuroprotetores e neurotóxicos de } \\
\text { andrógenos e estrógenos em um ambiente de estresse } \\
\text { oxidativo }\end{array}$ & Biology of Sex Differences & 6,2 & In vivo \\
\hline 2018 & YAO Q, et al. & $\begin{array}{l}\text { Efeitos da perda de hormônio ovariano nas placas } \\
\text { neuríticas e no fluxo autofágico no cérebro de } \\
\text { camundongos transgênicos duplos APP / PS1 fêmeas } \\
\text { adultas }\end{array}$ & $\begin{array}{c}\text { Acta Bioochimica et } \\
\text { biophysica sinica (ABBS) }\end{array}$ & 4,3 & In vivo \\
\hline 2016 & $\begin{array}{l}\text { WAHJOEPRAM } \\
\text { ANO EJ, et al. }\end{array}$ & $\begin{array}{l}\text { Os efeitos da suplementação de testosterona no } \\
\text { funcionamento cognitivo em homens mais velhos }\end{array}$ & $\begin{array}{l}\text { CNS Neurol Disord Drug } \\
\text { Targets }\end{array}$ & 6,7 & In vivo \\
\hline
\end{tabular}




\begin{tabular}{|c|c|c|c|c|c|}
\hline Ano & Autores & Título do artigo & Periódico & Fator de impacto & Tipo de experimento \\
\hline 2016 & $\begin{array}{l}\text { TSCHIFFELY } \\
\text { AE, et al. }\end{array}$ & $\begin{array}{l}\text { Uma avaliação comparativa de tratamentos com } 17 \beta \text { - } \\
\text { estradiol e seu pró-fármaco seletivo para o cérebro em } \\
\text { um modelo de camundongo duplo transgênico da doença } \\
\text { de Alzheimer }\end{array}$ & Horm Behav & 6,4 & In vivo \\
\hline 2017 & $\begin{array}{l}\text { TSCHIFFELY } \\
\text { AE, et al. }\end{array}$ & $\begin{array}{l}\text { Uma investigação exploratória do tratamento de } \\
\text { estrogênio seletivo para o cérebro em homens usando } \\
\text { um modelo de camundongo da doença de Alzheimer }\end{array}$ & Horm Behav & 6,4 & In vivo \\
\hline 2017 & $\begin{array}{l}\text { GIRARD R, } \\
\text { et al. }\end{array}$ & $\begin{array}{l}\text { A terapia hormonal no início da pós-menopausa aumenta } \\
\text { a atividade pré-frontal relacionada ao controle cognitivo }\end{array}$ & Scientific Reports & 7,1 & In vivo \\
\hline 2016 & $\begin{array}{l}\text { RETTBERG JR, } \\
\text { et al. }\end{array}$ & $\begin{array}{l}\text { Identificação de mulheres na pós-menopausa em risco } \\
\text { de declínio cognitivo em uma coorte saudável usando um } \\
\text { painel de indicadores metabólicos clínicos: Potencial } \\
\text { para detectar um fenótipo metabólico de risco de } \\
\text { Alzheimer }\end{array}$ & Neurobiol Aging & 8,0 & In vivo \\
\hline 2020 & $\begin{array}{l}\text { RAHMAN A, et } \\
\text { al. }\end{array}$ & $\begin{array}{l}\text { Modificadores do risco de Alzheimer impulsionados pelo } \\
\text { sexo: um estudo de imagem cerebral multimodal }\end{array}$ & Neurology & 9,5 & In vivo \\
\hline 2020 & $\begin{array}{l}\text { SUNDERMANN } \\
\text { EE, et al. }\end{array}$ & $\begin{array}{l}\text { Diferenças sexuais nos biomarcadores Tau relacionados } \\
\text { ao Alzheimer e um efeito mediador da testosterona }\end{array}$ & Biology of sex differences & 6,2 & $\begin{array}{l}\text { Dados extraídos do } \\
\text { ADNI dni.loni.usc.edu. }\end{array}$ \\
\hline 2020 & $\begin{array}{l}\text { CONNER MR, } \\
\text { et al. }\end{array}$ & $\begin{array}{l}\text { Impactos biológicos do sexo e dos hormônios sexuais } \\
\text { sobre os déficits na memória tipo episódica em um } \\
\text { modelo de rato de estágios precoces e pré-motores da } \\
\text { doença de Parkinson }\end{array}$ & Frontiers in Neurology & 4,03 & In vivo \\
\hline 2020 & $\begin{array}{l}\text { DE LANGE AG, } \\
\text { et al. }\end{array}$ & $\begin{array}{l}\text { Envelhecimento cerebral da mulher: efeitos da exposição } \\
\text { ao hormônio sexual, gravidez e risco genético para a } \\
\text { doença de Alzheimer }\end{array}$ & Human Brain Mapping & 8,3 & $\begin{array}{l}\text { Análise de dados } \\
\text { secundários }\end{array}$ \\
\hline
\end{tabular}

Fonte: Fonseca BS, et al., 2021. 


\section{DISCUSSÃO}

O processo de senescência fisiológico pode ser caracterizado pela redução de certas atividades cognitivas, como a memória. Na Doença de Alzheimer (DA) esse declínio cognitivo tende a ser mais significativo e com uma evolução mais rápida (WELLER J e BUDSON A, 2018). Estudos acerca da neuropatologia relacionada a DA relatam a deposição de placas formadas pela proteína beta-amiloide $(A \beta)$ e emaranhados neurofibrilares de proteína Tau hiperfosforilada no meio intracelular e extracelular, respectivamente. O aumento desses peptídeos leva a lesões neuronais, perda de sinapses e consequentemente um processo progressivo de neurotoxicidade (PIKE CJ, 2017; MUCKE L e SELKOE DJ, 2012). Apesar da influência desses peptídeos, entende-se que a etiologia da DA é multifatorial, envolvendo alteração ou predominância de genes, déficit hormonal, presença de radicais livres e acúmulo de proteínas específicas, Aß e Tau (DETURE MA e DICKSON DW, 2019; KHADILKAR SV e PATIL VA, 2019).

Identifica-se uma redução da atividade bioenergética e um aumento dos radicais livres nos estágios mais precoces da DA. Disfunções mitocondriais podem colaborar para o avanço da DA, visto que essas organelas exercem atividades essenciais para manter a demanda metabólica e energética das atividades celulares. As proteínas $A \beta$ e Tau demonstraram certo grau de toxicidade sobre a ação mitocondrial, causando redução na disponibilidade de energia na forma de trifosfato de adenosina (ATP) (GRIMM A, et al., 2016).

O estudo feito por Grimm A, et al. (2016) se apoia na Hipótese da Cascata Mitocondrial, sustentada pela ideia de que fatores genéticos, hereditários e ambientais influenciam nas alterações que a mitocôndria pode sofrer associada à idade. Mudanças mitocondriais influenciam os sinais de envelhecimento, por sua vez, fatores que relacionam a durabilidade dessas organelas podem estar veiculados a um processo de envelhecimento mais lento (SWERDLOW RH, et al., 2014).

Os resultados do estudo feito por Grimm A, et al. (2016) demostram um benefício da terapia com hormônios sexuais. A ação da testosterona sobre as células danificadas, principalmente pela deposição de proteína precursora amiloide (APP) / $A \beta$, promovendo um aumento bioenergético significativo na atividade celular. Além disso, o estrogênio e progesterona promoveram restauração da respiração máxima e da capacidade de reserva respiratória celular, que foram parâmetros bioenergéticos alterados pela proteína Tau mutante (GRIMM A, et al., 2016).

A DA é influenciada por certos fatores genéticos, um dos mais estudados é o alelo e4 do gene da ApoE. $A$ análise das diferenças entre homens e mulheres que possuem ApoE4, demonstram um aumento significativo de proteína Tau no sexo feminino (SUNDERMANN EE, et al., 2020).

Segundo o trabalho apresentado por Sundermann EE, et al. (2020), em que foi realizado reajustes de testosterona em homens e mulheres portadores de ApoE, foi identificado que baixos níveis de testosterona culminam em maior predisposição para a proteína Tau patológica. Sabe-se que no sexo feminino a presença de ApoE ocorre com mais frequência, além disso, o sexo é caracterizado por baixas concentrações de testosterona, resultando na presença da Tau patológica com maior frequência em mulheres.

A provável explicação para a menor prevalência do peptídeo Tau no sexo masculino está veiculada a ação neuroprotetora da testosterona, que promove ações antiinflamatórias, reduzindo o estresse oxidativo e promovendo um ambiente menos propício para deposição dos biomarcadores da DA (OTA H, et al.,2012), apoiando a hipótese proposta por essa revisão.

A exposição a estrogênios exógenos, como ocorre na terapia de reposição hormonal (TRH) iniciada na menopausa, demonstram efeitos positivos sobre o envelhecimento cerebral em portadoras de ApoE4 que realizam a TRH à base de estradiol precocemente, preferencialmente antes da menopausa. Contudo, contradizendo a hipótese defendida nessa revisão, esse mesmo estudo demonstrou que os esteroides sexuais, em determinadas situações, podem não comportar como hormônios neuroprotetores. Tal situação ocorre diante da TRH com estradiol em mulheres não portadoras de ApoE, onde não foi evidenciado benefícios relacionados ao envelhecimento cerebral. $O$ estudo ressalta ainda que o excesso dos hormônios sexuais, após a menopausa, pode atuar negativamente nas células neuronais. Pontua-se que essa pesquisa é de natureza transversal e para uma maior compreensão dos mecanismos envolvidos é necessário a realização de um estudo longitudinal (DE LANGE AG, et al., 2020). 
O plexo coroide localiza-se nos ventrículos cerebrais, realizando a produção e secreção do líquido cefalorraquidiano e de moléculas bioativas, como a $A \beta$ e outras proteínas responsáveis pelo seu processo de transporte e degradação. A apolipoproteina $J(A p o J)$ atua na degradação da $A \beta$, impedindo seu acúmulo no sistema nervoso central (SNC). Com o envelhecimento, ocorre uma redução mais significativa na expressão da ApoJ em mulheres em comparação aos homens. As alterações hormonais presentes no sexo feminino durante o processo de senescência podem justificar esses achados (DUARTE AC, et al., 2020).

As doenças neurodegenerativas possuem etiologia multifatorial. A presença de citocinas inflamatórias encontradas no SNC induz um estado de estresse oxidativo e aumento na expressão de determinadas proteínas encontradas na fisiopatologia das doenças neurodegenerativas, como a DA e demência de Parkinson (DP) (CONNER MR, et al., 2020).

A BACE-1, enzima responsável pela clivagem da APP no local $\beta$, tem a sua expressão aumentada em meio ao estresse oxidativo. Ademais, promove um aumento na geração de $A \beta$, contribuindo para patologia da DA. O trabalho realizado por Conner MR, et al. (2020) apresenta redução na expressão de BACE-1 com a exposição ao hormônio $17 \beta$-estradiol.

O SIRT-1 faz parte de um grupo de proteínas destinadas ao controle epigenético e pode atuar no mecanismo de neuroproteção contra neurotoxicidade presente em doenças neurodegenerativas. A ativação da SIRT-1 é exacerbada durante a exposição ao estradiol, promovendo uma redução no estresse oxidativo e na extensão do processo inflamatório do SNC. Dessa forma, essa proteína tem ação significativo na longevidade celular, inclusive das células nervosas, resultando em um controle do declínio cognitivo (CONNER MR, et al., 2020; KHAN M, et al., 2019).

As células do SNC podem atuar de diferentes formas tanto no processo de neurotoxicidade, quanto nos mecanismos de neuroproteção. O experimento realizado por Duong $P$, et al. (2020) expõe células neuronais e células da glia a um estresse oxidativo e posteriormente realizam a administração de estrogênio e testosterona no meio neurotóxico. O resultado obtido foi uma ação protetora dos hormônios sexuais nas células neuronais, reafirmando a hipótese defendida nessa revisão. Por outro lado, a ação dos esteroides sobre as células da glia não foram positivas, aumentando os danos oxidativos nas mesmas.

As células da glia possuem papel importante no SNC, fagocitando as células neuronais inviáveis ou marcadores inflamatórios presentes, mantendo um ambiente ideal para o bom funcionamento das atividades nervosas. O envelhecimento pode aumentar a suscetibilidade a processos inflamatórios, exigindo maior atuação das células gliais. Assim, mecanismos que conservassem a atividade das células gliais poderiam se comportar como protetores contra processos degenerativos do SNC. Os estudos de Duonng P, et al. (2020) mostram que isso não é possível com os esteroides sexuais.

Contradizendo o estudo proposto por Duonng P, et al. (2020), os resultados apresentados nas pesquisas de Honma N, et al. (2017) evidenciaram que a diferença do tecido cerebral de pessoas saudáveis e aquelas com DA foi justamente a redução da expressão de receptores de estrogênio nas células gliais. Foi proposto que a ausência da expressão desses receptores tornaria o indivíduo mais suscetível a DA, já que o hormônio sexual se comportaria como um neuroprotetor para célula glial (MA Y, et al., 2016).

O fator liberador de corticotrofina (CRF) controla mecanismos veiculados ao estresse celular, e alguns dos seus receptores é influenciado pelo sexo, logo podem se comportar de formas distintas em um ambiente estressor. Resultados expostos no trabalho realizado por Bangaser D, et al. (2017) demonstram que o sexo feminino tende a ter uma superexpressão do CRF, sendo que esse intensifica o estresse celular e promove maior fosforilação de BACE-1, provocando aumento na secreção de proteína $A \beta$. Essas particularidades biomoleculares podem justificar as diferenças sexuais na fisiopatologia da DA.

Como mencionado anteriormente, a fisiopatologia da DA é influenciada pelos estressores celulares e a apneia do sono está relacionada com eventos hipoxêmicos, que resultam em estresse oxidativo. Além disso, hipóxia crônica intermitente $(\mathrm{HCl})$ pode provocar uma redução na testosterona, principalmente no sexo masculino. Desse modo, presume-se que o efeito neuroprotetor desses hormônios também é reduzido (SNYDER B, et al., 2018; WAHJOEPRAMANO EJ, et al., 2016). Digno de nota, experimentos feitos em ratos 
machos gonadectomizados, que receberam suplemento de diidrotestosterona e possuíam $\mathrm{HCl}$, contraditoriamente tiveram algumas áreas cognitivas afetadas, mesmo diante a suplementação hormonal. Tal achado pode indicar a necessidade da sinalização estrogênica concomitante com a androgênica para reduzir os efeitos deletérios da apneia do sono (SNYDER B, et al., 2018).

Em estudo realizado com camundongos hAPP-J20 de DA, em que se relacionava as alterações do ciclo ovariano e a presença de alterações cognitivas, notou-se que nas fases em que havia o predomínio de estrogênio era identificado alteração na capacidade de aprendizagem e de memória, o que não era visto nos estágios com progesterone. A atuação negativa desses hormônios sobre regiões específicas do SNC, hipocampo e amígdala, coincidem com áreas que são acometidas de forma significativa na DA (BROESTL L, et al., 2018; YAO Q, et al., 2018).

Pesquisas realizadas com uma pró-droga seletiva de 17ß-estradiol no cérebro, 17ß-dihydroxyestra-1,4dien-3-one (DHED), demonstrou benefícios contra déficits neurobioquímicos. A administração de DHED resultou na limitação do transporte de APP para o processo de clivagem, promovendo um déficit na formação de $A \beta$ e avanços nos aspectos cognitivos. Esse benefício foi identificado nos casos de intervenções precoces, inclusive antes da menopausa. Digno de nota, não foi encontrado nenhum efeito sobre as fêmeas ovariectomizadas que receberam a terapia. A explicação para tal ocorrido pode ser relacionada com uma provável redução da APP/ A $\beta$ devido a cirurgia (TSCHIFFELY AE, et al., 2016; TSCHIFFELY AE, et al., 2017).

Tschiffely AE, et al. (2017) observou benefícios da terapia com DHED para o sexo masculino, assim como foi evidenciado no sexo feminino. No entanto, a terapia feita diretamente com $17 \beta$-estradiol não demonstrou eficácia nos homens, mas foi benéfico para mulheres.

Na DA a deposição de proteína $A \beta$ ocorre em diferentes regiões do SNC, principalmente no hipocampo e o córtex cerebral, dentre essas o lobo frontal é o mais estudado. Paciente com DA expressam menos receptores de estrogênio no córtex de tecidos cerebrais, e essa redução receptores estrogênicos pode estar relacionada com uma menor atuação do estrogênio no tecido cerebral, tornando viável a formação e deposição de placas (HONMA N, et al., 2017).

A avaliação da TRH em mulheres na faixa etária de 52 anos demonstrou um aumento significativo da atividade pré-frontal lateral, área associada com a DA, principalmente em estágios iniciais. Girard R, et al. (2017) realizou um experimento em que foi induzido a supressão dos hormônios ovarianos em mulheres mais jovens, observando uma redução da atividade de certas regiões cerebrais, como o cíngulo, córtex pré-frontal e giro frontal. Após a administração de estradiol foi observado as alterações nas regiões, notando um retorno das atividades a níveis normais.

A menopausa é considerada o momento da senescência reprodutiva da mulher, onde é caracterizada por uma alteração hormonal, tendendo para a redução dos esteroides sexuais. Sabe-se que a menarca tardia e a menopausa precoce oferecem um maior risco para o desenvolvimento da DA e, sugere-se, que o período de transição da menopausa pode ser um evento metabólico significativo para o aumento de deposição da proteína A (RAHMAN A, et al., 2020).

A HbA1c, HDL-colesterol e LDL-colesterol são alguns dos biomarcadores metabólico que sofrem alterações diretamente relacionadas com a idade. Apesar de serem biomarcadores periféricos eles podem indicar o estado do metabolismo de órgãos nobres, como o cérebro. Em um experimento realizado com mulheres, com o objetivo de identificar os benefícios da TRH sobre os biomarcadores do envelhecimento, foi observado uma melhora cognitiva significativa, além de aumento na $\mathrm{HbA1c}$ e no colesterol HDL e diminuição no LDL. No mesmo estudo, notou-se que as mulheres tendem a desenvolver mudanças nos biomarcadores mais precocemente que os homens, tendo a menopausa como um importante fator motivacional para essa diferença. Desta forma, o período de transição da menopausa pode influenciar no aumento dos biomarcadores da DA, as alterações metabólicas evidenciadas nessa fase podem estimular a deposição de proteínas $A \beta$, em algumas mulheres (RETTBERG JR, et al., 2017).

Por conseguinte, o estudo de Girard R, et al. (2017) relata o benefício da terapia hormonal precoce, assim como em outros artigos supracitados, iniciando antes da menopausa. No entanto, mulheres que iniciaram a terapia após a menopausa tiveram efeitos negativos sobre a cognição. 


\section{CONSIDERAÇÕES FINAIS}

Nesta revisão foram reunidas informações de alguns dos principais estudos que abordam as diferenças sexuais na DA, além dos benefícios que os hormônios sexuais exercem no sistema nervoso central, sendo um importante protetor contra os mecanismos que envolvem o envelhecimento. A partir da união dessas informações é possível presumir que a predisposição e fisiopatologia da DA cursa com algumas particularidades quanto ao sexo, levando em consideração alterações hormonais, como por exemplo as evidenciadas na menopausa. Ademais, as alterações nos esteroides sexuais podem levar a disfunções celulares e a deposição de proteínas específicas, como a $A \beta$ e a Tau, no SNS. Esses eventos resultam no declínio cognitivo progressivo, caracterizando a DA. Portanto, torna-se relevante avaliar essas diferenças a fim de implantar uma terapia eficaz e direcionada as particularidades de cada sexo, visando a possibilidade de prevenir ou reduzir os sinais/sintomas da DA.

\section{REFERÊNCIAS}

1. ALTMANN A, et al. Sex Modifies the APOE-Related Risk of Developing Alzheimer's Disease. Ann Neurol, 2014; 75(4): 563-573.

2. BANGASSER D, et al. Corticotropin-releasing factor overexpression gives rise to sex differences in Alzheimer's disease-related signaling. Mol Psychiatry, 2016; 22(8):1126-1133.

3. BRINTON RD, et al. Perimenopause s a neurological transition state. Nature Reviews Endocrinology, 2015; 11: 393405.

4. BROESTL L, et al. Ovarian Cycle Stages Modulate Alzheimer-Related Cognitive and Brain Network Alterations in Female Mice. Eneuro, 2018; 5(8) 0132-17.2018 1-8.

5. CONNER MR, et al. Biological Sex and Sex Hormone Impacts on Deficits in Episodic-Like Memory in a Rat Model of Early, Pre-motor Stages of Parkinson's Disease. Frontiers in Neurology, 2020; 11:942.

6. CUI J, et al. Estrogen synthesis and signaling pathways during aging: from periphery to brain. Trends Mol Med, 2013; 19(3): 197-209.

7. DE LANGE AG, et al. Women's brain aging: Effects of sex-hormone exposure, pregnancies, and genetic risk for Alzheimer's disease. Human Brain Mapping, 2020; 41(18):5141-5150.

8. DETURE MA, DICKSON DW. The neuropathological diagnosis of Alzheimer's disease. Mol Neurodegener, 2019; $14(1): 32$.

9. DUARTE AC, et al. Age, Sex Hormones, and Circadian Rhythm Regulate the Expression of Amyloid-Beta Scavengers at the Choroid Plexus. International Journal of Molecular Sciences, 2020; 21(18):6813.

10. DUONG P, et al. Neuroprotective and neurotoxic outcomes of androgens and estrogens in an oxidative stress environment. Biology of Sex Differences, 2020; 11:12.

11. GIRARD R, et al. Hormone therapy at early post-menopause increases cognitive control-related prefrontal activity. Scientific Reports, 2017; 7:44917.

12. GRIMM A, et al. Sex hormone-related neurosteroids differentially rescue bioenergetic deficits induced by amyloid- $\beta$ or hyperphosphorylated tau protein, Springer Link, 2016; 73(1):201-2015.

13. HONMA N, et al. Estrogen-Related Factors in the Frontal Lobe of Alzheimer's Disease Patients and Importance of Body Mass Index. Scientific Reports, 2017; 7:726.

14. KHADILKAR SV, PATIL VA. Sex Hormones and Cognition: Where Do We Stand? J Obstet Gynaecol India, 2019; 69(4):303-312.

15. KHAN M, et al. 17ß-Estradiol Modulates SIRT1 and Halts Oxidative Stress-Mediated Cognitive Impairment in a Male Aging Mouse Model. Cells, 2019; 8(8), 928.

16. MA Y, et al. Estrogen replacement therapy-induced neuroprotection against cerebral ischemia-reperfusion injury involves the activation of astrocytes via the $\beta$-estrogen receptor. Sci Rep, 2016; 6:21467.

17. MUCKE L, SELKOE DJ. Neurotoxicity of Amyloid $\beta$-Protein: Synaptic and Network Dysfunction. Cold Spring Harb Perspect Med, 2012; 2(7): a006338.

18. NICHOLS E, et al. Global, regional, and national burden of Alzheimer's disease and other dementias, 1990-2016: a systematic analysis for the Global Burden of DiseaseStudy 2016. The Lancet Neurology, 2019; 18:88-106.

19. OTA H, et al. Testosterone deficiency accelerates neuronal and vascular aging of SAMP8 mice: protective role of Enos and SIRT1. Plos One, 2012; 7(1): e29598.

20. PIKE CJ. Sex and the development of Alzheimer's disease. Journal of Internal Medicine, 2017; 95(1-2): 671-680.

21. PRINCE M, et al. The global prevalence of dementia: a systematic review and metaanalysis. Alzheimers Dement, 2013; $9(1): 63-75$. 
22. RAHMAN A, et al. Sex-driven modifiers of Alzheimer risk: A multimodality brain imaging study. Neurology, 2020; 95(2):e 166-178.

23. RETTBERG JR, et al. Identifying postmenopausal women at risk for cognitive decline within a healthy cohort using a panel of clinical metabolic indicators: potential for deecting an at-Alzheimer's risk metabolic phenotype. Neurobiol Aging, 2016; 40:155-163.

24. RODRIGUES TQ, et al. Impacto da Doença de Alzheimer na qualidade de vida de pessoas idosas: revisão de literatura. Revista Eletrônica Acervo Saúde, 2020; 12(4):e2823.

25. SCHUMACHER M, et al. Steroid hormones and neurosteroids in normal and pathological aging of the nervous system. Prog Neurobiol, 2003; 71(1):3-29.

26. SNYDER B, et al. Androgens modulate chronic intermittent hypoxia effects on brain and behavior. Horm Behav, 2018; 106: 62-73.

27. SUNDERMANN EE, et al. Sex differences in Alzheimer's-related Tau biomarkers and a mediating effect of testosterone. Biology of sex diferences, 2020; 11(1):33.

28. SWERDLOW RH, et al. The Alzheimer's disease mitochondrial cascade hypothesis: progress and perspectives. Biochim Biophys Acta, 2014; 1842(8): 1219-1231.

29. TEJEDOR NV, et al. Perivascular spaces are associated with tau pathophysiology and synaptic dysfunction in early Alzheimer's continuum. Alzheimer's Research \& Therapy, 2021; 13(135): 1-13.

30. TSCHIFFELY AE, et al. A comparative evaluation of treatments with $17 \beta$-estradiol and its brain-selective prodrug in a double-transgenic mouse model of Alzheimer's disease. Horm Behav, 2016; 83:39-44.

31. TSCHIFFELY AE, et al. An exploratory investigation of brain-selective estrogen treatment in males using a mouse model of Alzheimer's disease. Horm Behav, 2017; 98:16-21.

32. VAN DAM D, DE DEYN PP. Drug Discovery in dementia: the role of rodent models. Nat Rev Drug Discov, 2006; 5(11): 956-970.

33. WAHJOEPRAMONO EJ, et al. The effects of testosterone supplementation on cognitive functioning in older men. CNS Neurol Disord Drug Targets, 2016; 15(3):337-343.

34. WELLER J, BUDSON A. Current understanding of Alzheimer's disease diagnosis and treatment. F1000Res, 2018; 7(F1000Faculty Rev): 1161.

35. WORLD HEALTH ORGANIZATION (WHO). Global status report on the public health response to dementia. Geneva: World Health Organization. $2021 . \quad$ Disponível em: https://apps.who.int/iris/bitstream/handle/10665/344701/9789240033245-eng.pdf. Acesso em: 9 de setembro de 2021.

36. YAO Q, et al. Effects of ovarian hormone loss on neuritic plaques and autophagic flux in the brains of adult female APP/PS1 double-transgenic mice. Acta Bioochimica et biophysica sinica, 2018; 50(5), 447-455.

37. ZÁRATE S, et al. Role of Estrogen and Other Sex Hormones in Brain Aging. Neuroprotection and DNA Repair. Front Aging Neurosci, 2017; 9:430. 